\title{
A Method For Eclipsing Component Identification In Large Photometric Datasets
}

\author{
J. Devor (jdevor@cfa.harvard.edu) and D. Charbonneau \\ Harvard-Smithsonian Center for Astrophysics, 60 Garden St., Cambridge, MA \\ 02138, USA
}

\begin{abstract}
We describe an automated method for assigning the most likely physical parameters to the components of an eclipsing binary (EB), using only its photometric light curve and combined color. In traditional methods (e.g. WD and EBOP) one attempts to optimize a multi-parameter model over many iterations, so as to minimize the chi-squared value. We suggest an alternative method, where one selects pairs of coeval stars from a set of theoretical stellar models, and compares their simulated light curves and combined colors with the observations. This approach greatly reduces the EB parameter-space over which one needs to search, and allows one to determine the components' masses, radii and absolute magnitudes, without spectroscopic data. We have implemented this method in an automated program using published theoretical isochrones and limb-darkening coefficients. Since it is easy to automate, this method lends itself to systematic analyses of datasets consisting of photometric time series of large numbers of stars, such as those produced by OGLE, MACHO, TrES, HAT, and many others surveys.
\end{abstract}

\section{Introduction}

Eclipsing double-lined spectroscopic binaries provide the only method by which both the masses and radii of stars can be estimated without having to resolve spatially the binary or rely on astrophysical assumptions. Despite the large variety of models and parameter-fitting implementations (e.g. WD and EBOP), their underlying methodology is essentially the same. Photometric data provides the light curve of the EB, and spectroscopic data provide the radial velocities of its components. The depth and shape of the light curve eclipses constrain the components' brightness and fractional radii, while the radial velocity sets the length scale of the system. In order to characterize fully the components of the binary, one needs to combine all of this information. Unfortunately, only a small fraction of all binaries eclipse, and spectroscopy with sufficient resolution can be performed only for bright stars. The intersection of these two groups leaves a pitifully small number of stars.

In the past decade, there has been a dramatic growth in the number of stars with high-quality, multi-epoch, photometric data. This has been due to major advances in both CCD detectors and the implementation of image-difference analysis techniques (Crotts, 1992; Alard et al., 1998;

(c) 2018 Kluwer Academic Publishers. Printed in the Netherlands. 
Alard, 2000), which enables simultaneous photometric measurements of tens of thousands of stars in a single exposure. Today, there are many millions of light curves available from a variety of surveys, such as OGLE, Udalski et al. (1994); MACHO, Alcock et al. (1998); TrES, Alonso et al. (2004); and HAT, Bakos et al. (2004). But there has not been a corresponding growth in the quantity of spectroscopic data, nor is this likely to occur in the near future. Thus, the number of fullycharacterized EBs has not grown significantly. In recent years there has been a growing effort to mine the wealth of available photometric data, by employing simplified EB models in the absence of spectroscopic observations (Wyithe et al., 2001, 2002; Devor, 2004, 2005).

In this paper we present a novel approach, which utilizes theoretical models of stellar properties to estimate the orbital parameters as well as the masses, radii, and absolute magnitudes of the stars, while requiring only a photometric light curve and an estimate of the binary's combined color. This approach can be used to characterize quickly large numbers of eclipsing binaries, however it is not sufficient to improve stellar models since underlying isochrones must be assumed. We have created two implementations of this idea. The first, which we have named MECIexpress, and is described in section 2, is a "quick and dirty" program that is designed as a simple extension to the Detached Eclipsing Binary Light curve (DEBiL) fitter (Devor, 2004, 2005). The second, which we have named MECI, and is described in section 3, is considerably more accurate, but also more computationally demanding. The source code for both MECI-express and MECI will be provided upon request.

\section{Express Method for Eclipsing Component Identification (MECI-express)}

The primary application of MECI-express is to identify the stellar components of a given EB. It operates after a conventional EB modelfitting program has already analyzed the given EB's light curve. In our implementation, we chose to employ DEBiL (Devor, 2004, 2005) since it is simple, fast, and fully automated. The fitted parameters are the orbital period $(\mathrm{P})$, the apparent magnitudes $\left(\operatorname{mag}_{1,2}\right)$, and the fractional radii $\left(r_{1,2}\right)$ of the binary components. A fractional radius is defined as the radius $\left(R_{1,2}\right)$ divided by the sum of the components' semimajor axes (a). In MECI-express we iterate through a large group of MK spectral type pairings, to each of which we associate typical stellar parameters (Cox, 2000). These stellar parameters are the masses $\left(M_{1,2}\right)$, the radii $\left(R_{1,2}\right)$, and the absolute magnitudes $\left(M_{a g_{1,2}}\right)$ of the binary components. If the assumed values of the stellar parameters 
match the true values, then the stellar and fitted parameters should obey to the following equations:

$$
\begin{aligned}
\frac{4 \pi^{2} R_{1}^{3}}{G\left(M_{1}+M_{2}\right)} & =P^{2} r_{1}^{3} \\
\frac{4 \pi^{2} R_{2}^{3}}{G\left(M_{1}+M_{2}\right)} & =P^{2} r_{2}^{3} \\
M a g_{1}-M a g_{2} & =m a g_{1}-m a g_{2}
\end{aligned}
$$

We also may have additional constraints from the observed outof-eclipse combined colors of the system. For example, in the case of OGLE II targets, we have the estimated V-I color:

$$
M a g_{V}-M a g_{I}=\operatorname{mag}_{V}-\operatorname{mag}_{I}
$$

We assume that the color has been corrected for reddening and that no systematic errors are present, so any inequalities would be due to an incorrect choice for the component pairing. The likelihood of each pairing is assessed by calculating the difference between the lefthand-side (stellar parameters) and right-hand-side (fitted parameters) of each equation. These differences are divided by their uncertainties, and added in quadrature. The pairing with the smallest sum is deemed the most likely pairing. For each given EB light curve, MECI-express returns the list of the top ranked (most likely) binary pairings, with their corresponding sums. MECI-express can also be used to create a contour plot of the probability distribution for all pairings. We illustrate an example of individual MECI-express components in Figures 1.a-c, which are then combined to create the result shown in Figure 2.a.

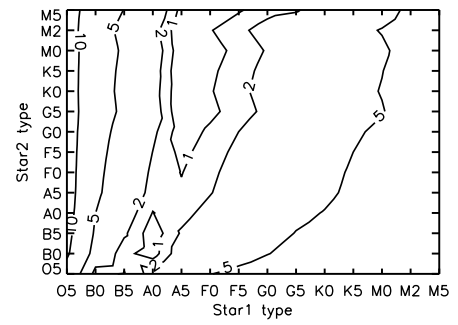

a. Constraints from (eq. 1)

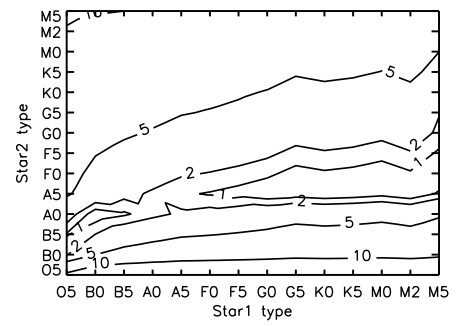

b. Constraints from (eq. 2)

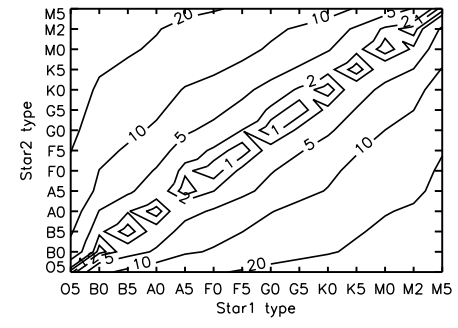

c. Constraints from (eq. 3)

Figure 1. Contour plots of the absolute difference between the left-hand-side and the right-hand-side of each equation, divided by its uncertainty, as applied to the WW Camelopardalis light curve (Lacy et al., 2002). Adding these results in quadrature, produces the likelihood plot shown in Figure 2.a. 


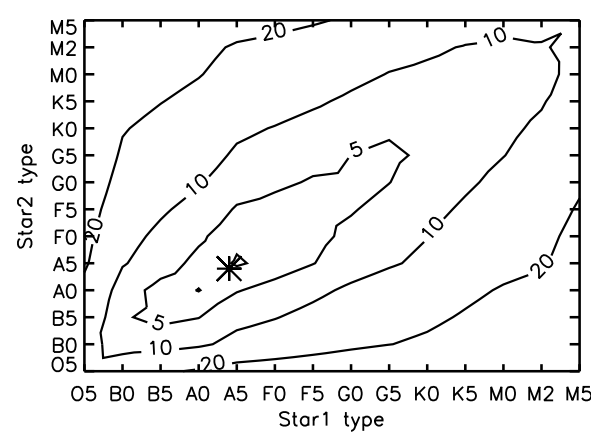

a. MECI-express likelihood plot

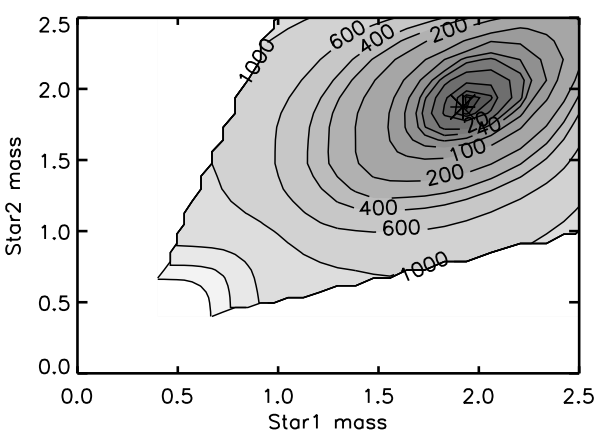

b. MECI likelihood plot (age $=0.6$ Gyr)

Figure 2. A comparison of the MECI-express (left) and MECI (right) likelihood contour plots for WW Camelopardalis. The value of the contours are described in the body of the text. The asterisk marks the solution of Lacy et al. (2002).

\section{Method for Eclipsing Component Identification (MECI)}

MECI was developed to improve significantly upon the accuracy of MECI-express (see Table 1). This was done as follows: We replaced the use of spectral types with the more fundamental (and continuous) quantities of mass and age. Furthermore, in MECI we assume that the two binary components are coeval, thus replacing the 2-dimensional spectral type - spectral type grid, with a 3-dimensional mass-mass-age grid. Finally, we no longer rely on parameter fits of the components' apparent magnitudes and fractional radii directly from the light curve, which are often very uncertain, nor do we assume constant limb-darkening coefficients. Instead, we interpolate these values for the given mass-mass-age pairing, from precalculated tables [Yonsei-Yale isochrones (Kim et al., 2002) ; ATLAS limb-darkening coefficients (Kurucz, 1992), used when $T_{\text {eff }} \geq 10000 K$ or $\log g \leq 3.5 ;$ PHOENIX limb-darkening coefficients (Claret, 1998, 2000), used when $T_{\text {eff }}<10000 K$ and $\left.\log g>3.5\right]$. Thus, by assuming the masses $\left(M_{1,2}\right)$ of the EB components and the system's age, we can look-up the radii $\left(R_{1,2}\right)$, the absolute magnitudes $\left(\operatorname{Mag}_{1,2}\right)$, and the limb-darkening coefficients for the binary components. We then use these values, as well as the observationally-determined period (P) and combined magnitude out of eclipse $\left(\operatorname{mag}_{\text {comb }}\right)$, to calculate the apparent magnitudes $\left(\operatorname{mag}_{1,2}\right)$ and factional radii $\left(r_{1,2}\right)$ of the EB components, as follows:

$$
\begin{aligned}
& \operatorname{mag}_{1}=\operatorname{mag}_{\mathrm{comb}}+2.5 \log \left[1+10^{-0.4\left(\mathrm{Mag}_{2}-\mathrm{Mag}_{1}\right)}\right] \\
& m a g_{2}=\operatorname{mag}_{1}+\left(\mathrm{Mag}_{2}-\mathrm{Mag}\right)_{1}
\end{aligned}
$$




$$
\begin{aligned}
a= & {\left[G\left(M_{1}+M_{2}\right)(P / 2 \pi)^{2}\right]^{1 / 3} \simeq } \\
& 4.206 R_{\odot}\left(M_{1} / M_{\odot}+M_{2} / M_{\odot}\right)^{1 / 3} P_{d a y}^{2 / 3} \\
r_{1,2}= & R_{1,2} / a
\end{aligned}
$$

Besides the epochs of eclipses, which can be determined directly from the EB light curve, there are only two additional parameters required for us to simulate the light curves of the given pairing: the orbital eccentricity (e) and inclination (i). For binaries with short periods $(\lesssim 2$ days $)$ and a secondary eclipse precisely half an orbit after the primary eclipse, it is reasonable to assume a circular orbit $(e=0)$. Otherwise, one should use the eccentricity derived by an EB modelfitting program (we use DEBiL). Finding the inclination robustly is more difficult. We employ a bracket search (Press et al., 1992), which returns the inclination that produces the best resulting fit.

To summarize, for every combination of component masses and system age of an EB, we can look-up, calculate, or fit all the parameters needed to simulate its light curve $(\mathrm{P}$, limb-darkening coefficients, $\operatorname{mag}_{1,2}, \mathrm{r}_{1,2}$, epochs of eclipses, e, i), as well as its apparent combined color. We systematically iterate through many such combinations. For each one we compare the expected light curve with the observations, and calculate the reduced chi-squared value $\left(\chi_{\nu}^{2}\right)$. We also compare each observed color $\left(O_{c} \pm \epsilon_{c}\right)$ with its calculated value $\left(C_{c}\right)$, and combine them by defining: score $\equiv\left(w \chi_{\nu}^{2}+\sum_{c=1}^{N}\left[\left(O_{c}-C_{c}\right) / \epsilon_{c}\right]^{2}\right) /(w+N)$. Where $w$ is the $\chi_{\nu}^{2}$ information weighting. We use $w=1$, and assume that the smaller the score, the more likely it is that we have chosen the correct binary pairing. One can visualized this result using a series of $\operatorname{score}\left(M_{1}, M_{2}\right)$ contour plots, each with a constant age (e.g. Figure 2.b).

\section{Conclusions}

We have described a novel method for identifying an EB's components using only its photometric light curve and combined color. By utilizing theoretical isochrones and limb-darkening coefficients, this method greatly reduces the EB parameter-space over which one needs to search. This approach seeks to estimate the masses, radii and absolute magnitudes of the components, without spectroscopic data. We described two implementations of this method, MECI-express and MECI, which enable systematic analyses of datasets consisting of photometric time series of large numbers of stars, such as those produced by OGLE, MACHO, TrES, HAT, and many others. Such techniques are expected to grow in importance with the next generation surveys, such as PanSTARRS (Kaiser et al., 2002) and LSST (Tyson, 2002). 
Table I. A comparison of the results produced by MECI-express, MECI, and conventional analyses with their uncertainties (Lacy et al., 2000, 2002, 2003). The square brackets with numerical values indicate the deviation of our results from those of the conventional approach.

\begin{tabular}{c|cc|ccc|ccc}
\hline \multirow{4}{*}{ Parameter } & \multicolumn{2}{|c|}{ MECI-express } & \multicolumn{3}{|c|}{ MECI } & \multicolumn{2}{|c}{ Lacy et al. (2000, 2002, 2003) } \\
& Mass 1 & Mass 2 & Mass 1 & Mass 2 & Age & Mass 1 & Mass 2 & Age \\
& {$\left[M_{\odot}\right]$} & {$\left[M_{\odot}\right]$} & {$\left[M_{\odot}\right]$} & {$\left[M_{\odot}\right]$} & {$[\mathrm{Gyr}]$} & {$\left[M_{\odot}\right]$} & {$\left[M_{\odot}\right]$} & {$[\mathrm{Gyr}]$} \\
\hline \multirow{3}{*}{ FS Mon } & $2.9(\mathrm{~A} 0)$ & $2.0(\mathrm{~A} 5)$ & 1.62 & 1.52 & 1.4 & 1.632 & 1.462 & 1.6 \\
& {$[77.7 \%]$} & {$[36.8 \%]$} & {$[0.6 \%]$} & {$[4.1 \%]$} & {$[0.2]$} & \pm 0.012 & \pm 0.010 & \pm 0.3 \\
WW Cam & $2.0(\mathrm{~A} 5)$ & $2.0(\mathrm{~A} 5)$ & 1.97 & 1.89 & 0.5 & 1.920 & 1.873 & 0.5 \\
& {$[4.2 \%]$} & {$[6.8 \%]$} & {$[2.8 \%]$} & {$[1.0 \%]$} & {$[0.0]$} & \pm 0.013 & \pm 0.018 & \pm 0.1 \\
BP Vul & $2.0(\mathrm{~A} 5)$ & $1.6(\mathrm{~F} 0)$ & 1.77 & 1.48 & 0.7 & 1.737 & 1.408 & 1.0 \\
& {$[15.1 \%]$} & {$[13.6 \%]$} & {$[2.1 \%]$} & {$[5.4 \%]$} & {$[0.3]$} & \pm 0.015 & \pm 0.009 & \pm 0.2 \\
\hline
\end{tabular}

We are grateful to Guillermo Torres for many helpful conversations.

\section{References}

Alard, C. \& Lupton, R. H. 1998, ApJ, 503, 325

Alard, C. 2000, A\&AS, 144, 363

Alcock, C., et al. 1998, ApJ, 492, 190

Alonso, R., et al. 2004, ApJ, 613, L153

Bakos, G., Noyes, R. W., Kovács, G., Stanek, K. Z., Sasselov, D. D., \& Domsa, I. 2004, PASP, 116, 266

Claret, A. 1998, A\&A, 335, 647

Claret, A. 2000, A\&A, 363, 1081

Cox, A. N. 2000, Allen's astrophysical quantities, (4th ed.; New York: AIP Press; Springer)

Crotts, A. P. S. 1992, ApJ, 399, L43

Devor, J. 2004, American Astronomical Society Meeting Abstracts, 205

Devor, J. 2005, ApJ, 628, 411

Kaiser, N., et al. 2002, SPIE, 4836, 154

Kim, Y., Demarque, P., Yi, S. K., \& Alexander, D. R. 2002, ApJS, 143, 499

Kurucz, R. L. 1992, IAU Symp. 149: The Stellar Populations of Galaxies, 149, 225

Lacy, C. H. S., Torres, G., Claret, A., Stefanik, R. P., Latham, D. W., \& Sabby, J. A. 2000, AJ, 119, 1389

Lacy, C. H. S., Torres, G., Claret, A., \& Sabby, J. A. 2002, AJ, 123, 1013

Lacy, C. H. S., Torres, G., Claret, A., \& Sabby, J. A. 2003, AJ, 126, 1905

Press, W. H., Teukolsky, S. A., Vetterling, W. T., \& Flannery, B. P. 1992, (2nd ed.; Cambridge: University Press)

Tyson, J. A. 2002, SPIE, 4836, 10

Udalski, A., et al. 1994, Acta Astronomica, 44, 165

Wyithe, J. S. B. \& Wilson, R. E. 2001, ApJ, 559, 260

Wyithe, J. S. B. \& Wilson, R. E. 2002, ApJ, 571, 293 